\title{
Tailoring Cell Behavior on Polymers by the Incorporation of Titanium Doped Phosphate Glass Filler**
}

\author{
By Wojciech Chrzanowski, Ensanya A. Abou Neel, Koon-Y. Lee, \\ Alexander Bismarck, Anne M. Young, Andrew D. Hart, Matthew J. Dalby \\ and Jonathan C. Knowles*
}

Understanding tissue response to materials, to enable modulation and guided tissue regeneration is one of the main challenges in biomaterials science. Nowadays polymers, glasses, and metals dominate as biomaterials. Often native properties of those materials are not sufficient and there is a need to combine them, so as to modify and adjust their properties to the application. The primary aim of this study was to improve cell response to polymer (PLDL) using phosphate glass as filler (titanium doped phosphate glass). As a control $\beta$-tricalcium phosphate (TCP) filler was used. Various concentrations of the filler were used (10-40 vol\%). Wetting behavior, $\zeta$-potentials, mechanical and thermal properties, and human cells response to the materials were evaluated. Results showed that with increase in glass filler loading wettability improved, $\zeta$-potentials dropped, and increase in stiffness of materials was observed. Importantly cell culture experiments showed more developed and well spread cells on the samples with glass content up to $20 \mathrm{vol} \%$. Cells responded much more positively to the glass filled samples than to TCP filled. However, expression of osteocalcin and osteopontin, proteins that indicate formation of the mineralized structures was positive for all the samples including pure PLDL. It was concluded that due to improved wetting behavior, lower $\zeta$-potentials, and specific chemistry of the glass filler it was possible to alter cells response, improve bioactivity of the polymer, and vary mechanical properties.

According to the most recent definition proposed by Ratner (unpublished), biocompatibility is the ability of materials to locally trigger and guide normal wound healing, reconstruction, and tissue regeneration. Moreover, Ratner

[*] Prof. J. C. Knowles, Dr. E. A. Abou Neel, Dr. A. M. Young UCL Eastman Dental Institute

256 Gray's Inn Road, London WC1X8LD, UK

E-mail: j.knowles@eastman.ucl.ac.uk

Dr. W. Chrzanowski

The Faculty of Pharmacy Pharmacy and Bank Building University of Sydney NSW 2006 Sydney Australia

Dr. W. Chrzanowski

Department of Mechanical Engineering James Watt South Building University of Glasgow

Glasgow G12 8QQ UK

Dr. K.-Y. Lee, Prof. A. Bismarck

Department of Chemical Engineering Polymer and Composite Engineering (PaCE) Group Imperial College London

South Kensington Campus London SW7 2AZ UK

A. D. Hart, Dr. M. J. Dalby

Centre for Cell Engineering Joseph Black Building University

of Glasgow Glasgow G12 8QQ UK introduced a new term called "biotolerability", which is defined as the ability of the materials to reside in the body for long periods of time with low degree of inflammatory reaction. Amongst various materials used to produce

Prof. J. C. Knowles

WCU Research Centre of Nanobiomedical Science

Dankook University, San\#29, Anseo-dong, Dongnam-gu

Cheonan-si, Chungnam, 330-714, South Korea

Dr. W. Chrzanowski

The Faculty of Pharmacy, University of Sydney

Pharmacy and Bank Building A15

Sydney, NSW 2006, Australia

[**] Acknowledgements, Authors would like to thank Purac Biomaterilas for providing the polymer, Plasma Biotal for providing tricalcium phosphate, and Dr. Mathis Riehle for the access to cell culture facilities. This work was supported in part by WCU Program through the National Research Foundation of Korea (NRF) funded by the Ministry of Education, Science and Technology (No. R31-10069). 
implants or tissue engineering devices, which are suppose to satisfy these definitions, polymers, ceramics, and glasses or combination of those materials ${ }^{[1-7]}$ are the most popular. Some of these materials are very well established and commonly used to produce various medical devices. Nevertheless, there is still a need for improvement of these materials and adjustment of their properties for specific applications e.g., directing and modulating cells growth, or inhibiting biofilm formation. Some of these materials are particularly suitable for advanced technologies related to drug, genes and growth factors delivery, bone cements and adhesives, degradable and bioresponsive implants and coatings. ${ }^{[8-16]}$ The main role of the materials is to support tissue regeneration, trigger, and/or inhibit particular biological or microbiological activity. It is well known that this can be achieved using chemical (Ca, $\mathrm{P}, \mathrm{Zn}, \mathrm{Mg}$ ), structural (including topographical), or mechanical cues of the materials such as stiffness. Often it is necessary to compromise mechanical, structural, and chemical properties to achieve "optimum" body response. Tailoring of these properties can be achieved by combining different types of materials. This approach has been developed for decades with material blends, composites, and filled materials now commonly used. $^{[17,18]}$ Concentrating on bone tissue applications and considering polymer based materials (filled polymers and composites), hydroxyapatite, tricalcium phosphate (TCP) or Bioglass are the most popular used fillers. ${ }^{[16,19-26]}$ Other interesting materials are phosphate glasses, which have readily tuneable properties to improve cellular activity ${ }^{[27-31]}$ or inhibit biofilm formation (antimicrobial glasses). ${ }^{[32-34]}$ To date they were rarely used as polymer fillers. Glass fibers reinforced polymers have been shown to have highly desirable both biological and mechanical properties, therefore glass fillers as a powder/particles seem to be alternative for other used fillers. The main advantage of these materials when used as filler is that their chemistry can be fine tuned. This providesan opportunity to modulate cell behavior via changes in the chemistry (glass co-doped with $\mathrm{Ti}, \mathrm{Zn}, \mathrm{Sr}$ ), degradation rate thus delivery of vital ions such as $\mathrm{P}$ and $\mathrm{Ca}$, $\mathrm{Mg}$ or $\mathrm{Fe}$, and finally change local micromechanical environment, which in turn impacts cells response. Because fillers change local and global mechanical properties of the material all the considerations of combined materials (polymer-filler) should include mechanical aspects and should relate them to the final tissue response.

For these reasons phosphate glass materials/fillers may be considered as an easy route to trigger and guide tissue regeneration or other biological/microbiological reaction. To date, influence of the particles morphology and size, chemical structure and degradation, changes to the local micromechanical environment on cell behavior has not been fully investigated. Typically changes to one of parameter causes changes to the others and this makes studies very complex. For this reason approach where influence of chosen parameters is tested separately with gradual increase in number of variables need to be employed.
To date, it was demonstrated that some phosphate glasses in combination with some polymers may be particularly useful to devise membranes or tooth fillings. ${ }^{[24,35-40]}$ It is envisaged that phosphate glasses in various forms may be applicable for load bearing implants (fiber reinforced composite materials - under development; data unpublished), wound healing dressings (antimicrobial activity) or bioadesives. ${ }^{[16]}$

Referring to the definitions presented by Ratner and literature review, the use of doped phosphate glasses in combination with other materials seems to be particularly relevant for biomaterials especially for bone tissue application (implants, coatings). Our previous studies have shown that phosphate glasses doped with titanium have improved significantly cells response. ${ }^{[27-29,41]}$ For this reason authors challenged to modulate cells behavior on polymers (PLDL) using glass filler (titanium/phosphate glass). It is hypothesised that using titanium doped phosphate glasses as a component (filler) of polymer based devices it is possible to improve cells activity and trigger osteoblastic differentiation ensuring low inflammatory reaction for bone tissue regeneration devices (e.g., implants, membranes, coatings). This improvement is a result of specific, tunable chemistry and degradation of the filler. It is also believed that biological or microbiological activity of such materials would improve in comparison with commonly used fillers due to their specific chemical structure.

The main objectives of this study were:

- to fabricate filled biodegradable polymer with devised phosphate glass to improve cell response,

- to investigate biocompatibility of the titanium doped phosphate glass filled polymer,

- to look into the effect of the filler on mechanical properties of the polyme

\section{Materials and Methods}

Materials

To fabricate a filled polymer two main components were used: filler and polymer.

Filler: Previous authors' studies showed that $5 \mathrm{~mol} \%$ titanium doped phosphate glass would be particularly suitable to modulate cells response. ${ }^{[27-29,41]}$ Therefore as primary filler this type of glass was used. To produce the glass powder of given composition-50 mol\% $\mathrm{P}_{2} \mathrm{O}_{5} 30 \mathrm{~mol} \% \mathrm{CaO}$ $15 \mathrm{~mol} \% \mathrm{Na}_{2} \mathrm{O} 5 \mathrm{~mol} \% \mathrm{TiO}_{2}$ - in the first instance glass was produced using following precursors: sodium dihydrogen orthophosphate $\left(\mathrm{NaH}_{2} \mathrm{PO}_{4}\right)$, calcium carbonate $\left(\mathrm{CaCO}_{3}\right)$, phosphorus pentoxide $\left(\mathrm{P}_{2} \mathrm{O}_{5}\right)$, and titanium dioxide $\left(\mathrm{TiO}_{2}\right)$ (BDH, Poole, UK). The glass was prepared by a conventional melt-quenching process by melting at $1300{ }^{\circ} \mathrm{C}$ for $3 \mathrm{~h}$. The melted glass was left to cool to room temperature and then ground and milled using agate mill to size $12 \mu \mathrm{m}$. The titanium doped phosphate glass powder (filler) was coded in the paper as "T5" filler.

As a control filler sintered $\beta$-TCP(Plasma-Biotal, UK) of average particle size $12 \mu \mathrm{m}$ was used; coded as "TCP" filler. 
Polymer: PURASORB PLDL 7038 (Purac, The Netherlands) copolymer of L-lactide and DL-lactide in a 70/30 molar ratio and with an inherent viscosity midpoint of $3.8 \mathrm{dl} \mathrm{g}^{-1}$ was used in the study.

\section{Methods of Materials Production}

Filled polymer samples were produced using solvent casting approach. In the first instance the glass suspension in polymer was prepared; the glass powder was added to the solvent (acetone) and stirred for $24 \mathrm{~h}$. Next PURASORB granules were gradually added to the suspension and stirred for another $24 \mathrm{~h}$, polymer weight to solvent volume ratio was $2 \%$ (wt./vol.). To ensure homogenous distribution of the filler, the polymer solution was sonicated for $5 \mathrm{~min}$. Polymer/glass suspension was then cast into petri dishes and left partly covered at room temperature to allow solvent evaporation. The samples were then separated from the petri dish and dried out under vacuum for $24 \mathrm{~h}$ at $60^{\circ} \mathrm{C}$, weight of $10 \mathrm{~N}$ was applied to the samples to prevent bending and remove residual stresses. Samples with 10, 20, and $40 \mathrm{vol} \%$ of the glass were produced and coded, respectively, PLDL-10\%T5, PLDL-20\%T5, and PLDL-40\%T5. Sample with $10 \mathrm{vol} \%$ of $\beta$-TCP was used as a control-PLDL-10\% TCP.

All studies were carried out on the side that had direct contact with the bottom of the petri dishes; it was assumed that due to evaporation conditions the top part of the samples may be covered with thin polymer layer that coat glass or TCP particles. This in turn would result in testing only the polymer (until polymer degrade and particles are uncovered) when top part was considered.

\section{Examinations}

\section{Scanning Electron Microscopy (SEM) Morphology and Filler Distribution Evaluation}

To evaluate the morphology of the fillers and their distribution in the polymer matrix, samples were mounted on carbon sticky tabs placed onto an aluminum pin stub (Agar Scientific, Stansted, UK). All polymeric specimens were gold coated (Quorum Technology, Newhaven, UK) and examined in a Cambridge 90B SEM (Cambridge Microscopes, Cambridge, UK) operating at 5 or $10 \mathrm{kV}$.

Wetting Behavior of (Titanium Doped) Phosphate Glass PLDL Composites

To determine the wettability of PLDL and its (titanium doped) phosphate glass PLDL, advancing water contact angle measurements were conducted using the sessile drop approach utilizing a DSA 10 (Krüss GmbH, Hamburg, Germany). Droplets of approximately $20 \mu \mathrm{l}$ of ultra pure water were placed on the samples surfaces using a motorized syringe and the drop volume was steadily

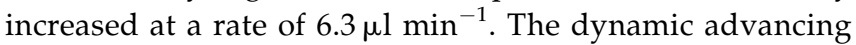
contact angle, i.e., the contact angle formed during the wetting process, was measured. The measurements were repeated at least five times on five different spots of a polymer (composite) film.

\section{Surface Characteristics of Titanium Doped Phosphate Glass/ PLDL composites}

The $\zeta$-potential of unmodified PLDL films, PLDL filled with glass $(10,20$, or $40 \mathrm{vol} \%)$ and $\beta$-TCP $(10 \mathrm{vol} \%)$ was measured using the Electrokinetic Analyzer (EKA; Anton Paar KG, Graz, Austria) based on the streaming potential method. ${ }^{[26]}$ To eliminate any errors that could be caused by the swelling of the polymer, the $\zeta$-potential as function time, $\zeta=f(\mathrm{t})$, was recorded at constant ionic strength $(1 \mathrm{mM} \mathrm{KCl})$ prior to measuring $\zeta=f(\mathrm{pH})$ in a $1 \mathrm{mM} \mathrm{KCl}$ supporting electrolyte solution. The streaming potential was generated by applying a steadily increasing pressure difference $(\Delta p)$ from 30 to 250 mbar across a channel which was created by stacking a PTFE film with a preformed channel between two sample films. The $\mathrm{pH}$ was controlled by adding either $0.1 \mathrm{M} \mathrm{HCl}$ or $0.1 \mathrm{M}$ $\mathrm{KOH}$ using a remote titration unit (RTU, Anton Paar KG, Graz, Austria) at a constant temperature of $20^{\circ} \mathrm{C}$.

\section{Mechanical Properties of Titanium Doped Phosphate Glass/ PLDL Composites}

Tensile strength and Young's modulus were measured on a dynamic mechanical analyzer (Perkin-Elmer DMA-7e, USA) at room temperature. The samples were mounted on the tangential clamp extension analysis measuring system. The dimensions of each specimen were measured with a micrometer. The initial applied load was $1 \mathrm{mN}$, and the specimen was tested in tension until failure at a loading rate of $200 \mathrm{mN} \mathrm{min}{ }^{-1}$. The stress-strain relationship was obtained, and the software (Pyris version 5 software, Perkin-Elmer, UK) also calculated the corresponding Young's modulus as an indicator of the material elasticity and tensile strength as the average of ultimate stress at the breaking point of the film.

\section{Differential Scanning Calorimetry (DSC) Glass Transition Temperature}

A sample of each of the specimens were prepared and placed into sample holder. The glass transition temperature $\left(T_{\mathrm{g}}\right)$ was determined using a Pyris Diamond DSC (Perkin-Elmer Instruments, USA). The instrument was calibrated using indium and zinc as standards. Samples $(n=3)$ were heated, cooled, and reheated from 25 to $250{ }^{\circ} \mathrm{C}$ at $50{ }^{\circ} \mathrm{C} \mathrm{min}{ }^{-1}$. $T_{\mathrm{g}}$ was calculated by the onset of change in the endothermic direction (upwards) of the heat flow of the second heating ramp. All tests were carried out under nitrogen purge.

\section{Cell Culture-Cytoskeleton, Differentiation}

In these experiments, human primary osteoblasts cells were seeded on the samples with density $10^{4}$ cells/sample and incubated with growth medium [Modified Eagles Medium ( $\alpha$-MEM Gibco), $10 \%$ fetal calf serum, and $1 \%$ penicillin and 


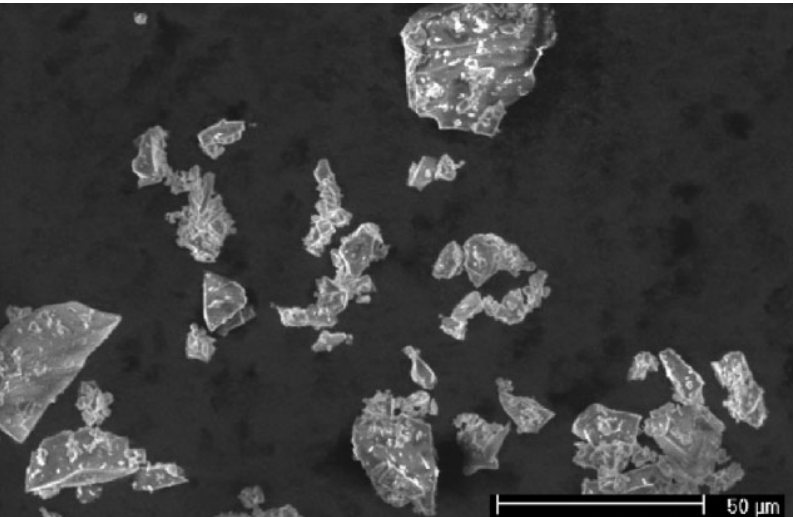

Fig. 1. SEM images of the glass powder. Images show angular shapes and non uniform size of the powder particles.

streptomycin solution] at $37^{\circ} \mathrm{C}$ in a humidified atmosphere of $5 \% \mathrm{CO}_{2}$ in air

Prior the test, samples were sterilized in ethanol for $1 \mathrm{~h}$ and washed with Hepes Saline (HS, SigmaAldrich) and ultrapure sterilized water. Tests were conducted in triplicate. Thermanox (TX) was used as positive controls.

To assess formation and organization of the cytoskeleton (immunofluorescence), cells were cultured for 3 days (early time point) and stained for actin (Rhodamine phalloidin), tubulin (anti-tubulin), and vinculin (anti-vinculin). Actin, which is a major structural protein, gives information on the general condition of the cells-ability to adhere and spread. ${ }^{[42]}$ Tubulin is another structural protein and is important in cell metabolism as vesicles are moved in and out of the cell (endocytosis and exocytosis) along the tubulin microtubules. ${ }^{[42,43]}$ While vinculin, is a protein involved in cell adhesion and shows contacts of the cells with materials. ${ }^{[4]}$ These adhesion points are important in cell signaling, proliferation, and differentiation.

To asses differentiation and maturation of the cells second group of samples were cultured for 21 days when osteoblast cells expected to start mineralization (if differentiation is triggered/enhanced by the substrate) by secreting bone specific extracellular matrix proteins [osteocalcin (OC) and osteopontin (OPN); immunofluorescence). Media was replaced every 3 days. At 21st day (late time point) cells were fixed with glutaraldehyde and stained for osteocalcin and osteopontin. Therefore, positive staining is an indication whether cells would be able to mineralize on the surfaces.

Both groups of samples were examined using fluorescent microscope (Zeiss, Axiovert 200M).

Results

Examinations

Scanning Electron Microscopy (SEM) - Morphology and Filler Distribution Evaluation

SEM observation of the T5 filler evidenced that the size of the powder is not uniform and varied between 4 and $90 \mu \mathrm{m}$. The particles had angular shape (Fig. 1). For the T5 filled polymer samples it was observed that for the lowest powder concentration $(10 \mathrm{vol} \%)$ the filler distribution was not uniform and some areas where powder was not present were observed (Fig. 2). Uniformity of distribution improved with increase in the filler content. Filler in both 20 and $40 \mathrm{vol} \%$ samples (Fig. 3), was uniformly distributed in the polymer structure. Visual assessment of the filler incorporation within the polymer revealed that in general the powder was well

a)

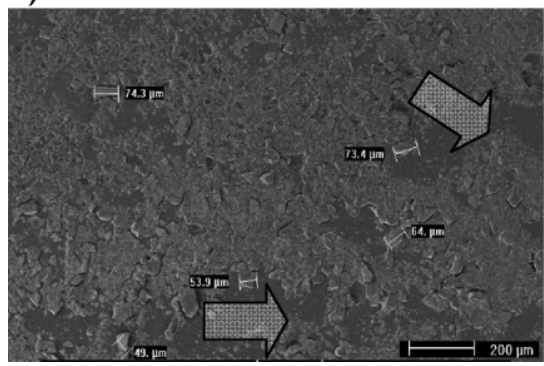

Fig. 2. SEM images of the polymer with $10 \mathrm{vol} \%$ of glass filler: (a) visible non uniform distribution of the particles; area with visual lack of the filler (arrow), (b) high magnification image shows good integration of the powder in the polymer structure; polymer filled well spaces between particles.

a)

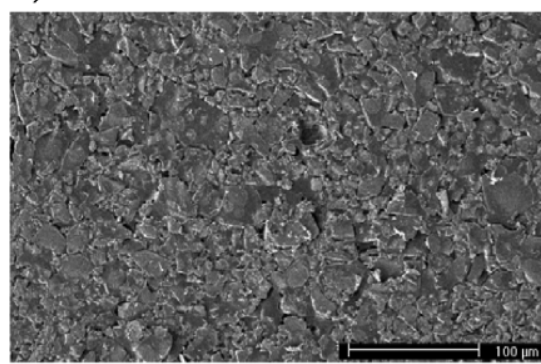

b)

C)

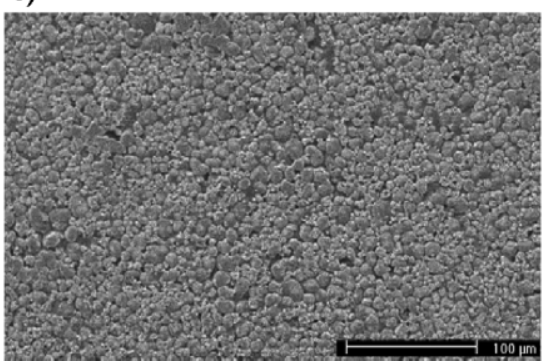

Fig. 3. SEM images of the polymer with (a) $20 \mathrm{vol} \%$ and (b) $40 \mathrm{vol} \%$ of glass filler, and (c) $10 \mathrm{vol} \%$ of $\beta$-TCP showing uniform distribution of the particles and good integration of the powders in the polymer structure. 
integrated with PLDL. Despite of good integration of the particles in the polymer matrix, some voids were only observed for $10 \mathrm{vol} \%$ of the glass filler [Fig. 2(b)].

TCP filler was average of $12 \mu \mathrm{m}$ (producer data), had angular shape and its distribution in the polymer was very uniform.

\section{Wetting Behavior of (Titanium Doped) Phosphate Glass PLDL Composites}

Assessment of the contact angle, which is a measure of the wettability of the surface, showed differences between the samples with different content of the filler when compared with pure PLDL samples (Table 1). PLDL, is essentially PDLLA, and rather hydrophobic with an advancing contact angle of about $80^{\circ}$, which is in good agreement with contact angles reported for PDLLA. ${ }^{[45]}$ Addition of 10 vol\% of TCP does not significantly affect the wetting behavior, although the average contact angle increases slightly. However, the addition of the ground titanium doped phosphate glass filler did result in a significant improvement of the wetting behavior, i.e., the advancing contact angle dropped by $10^{\circ}$. A further increase in the T5 filler content also resulted in a further significant reduction of the dynamic advancing contact angle. Moreover, once the advancing motion, i.e., the addition of water to the droplet was stopped, we observed that the water from the droplet wicked into the PLDL composites containing 20 and $40 \mathrm{vol} \%$ titanium doped phosphate glass. The higher the glass loading fraction the faster the water wicked into the sample. For instance, the water contact angle for the composite containing $40 \mathrm{vol} \%$ of the T5 filler rapidly decreased to 0 after about $15 \mathrm{~s}$ after stopping the addition of water into the droplet. Water wicked rapidly into the composites either through the gaps between the polymer and the glass filler [see SEM images Fig. 3 (a) and (b)]or into agglomerates of individual glass particles which are not fully impregnated by polymer. The wicking into the composite is primarily driven by the higher wettability of the glass filler present in the composite. However, one should note that the advancing wetting behavior of the composites is determined by the low energy part, i.e., the polymer, of the surface.

\section{Surface Characteristics of Titanium Doped Phosphate Glass PLDL Composites}

Measured $\zeta$-potentials reflect the formation of the electrochemical double layer, which is not only determined by the type and concentration of ions in the supporting electrolyte but

Table 1. Advancing water contact angle $\theta$, isoelectric point (iep) and $\zeta$-potential plateau ( $\zeta_{\text {plateau }}$ ) value at $\mathrm{pH}>6.5$.

\begin{tabular}{lccc} 
Sample & $\theta{ }^{\circ}{ }^{\circ}$ & iep $[\mathrm{pH}]$ & $\zeta_{\text {plateau }}[\mathrm{mV}]$ \\
\hline PLDL & $81.2 \pm 1.2$ & $4.0 \pm 0.1$ & $-30.2 \pm 0.6$ \\
PLDL-10\%TCP & $86.9 \pm 2.9$ & $3.7 \pm 0.1$ & $-23.9 \pm 0.7$ \\
PLDL-10\%T5 & $70.2 \pm 1.7$ & - & $-18.6 \pm 1.1$ \\
PLDL-20\%T5 & $57.8 \pm 2.1$ & - & $-23.5 \pm 1.3$ \\
PLDL-40\%T5 & $50.4 \pm 3.4$ & - & $-33.9 \pm 2.0$
\end{tabular}

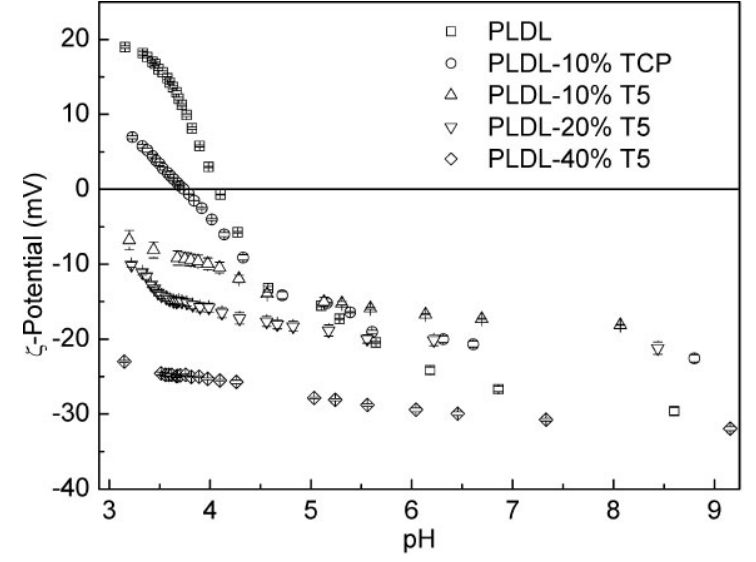

Fig. 4. $\zeta$-potential as function of $\mathrm{pH}$ measured in $1 \mathrm{mM} \mathrm{KCl}$ supporting electrolyte of PLDL and $\beta-T C P$ and ground titanium doped phosphate glass PLDL composites.

also by the presence of dissociable (Brønsted acidic or basic) functional groups on the materials surface and the hydrophilicity/phobicity of the material as well as the exposure time to the aqueous electrolyte. The $\zeta=f(\mathrm{pH})$ courses of PLDL and the composites, shown in Figure 4, are characteristic for materials containing Brønsted acidic surface groups having a low isoelectric point (iep), where $\zeta=0$, and plateau area $\left(\zeta_{\text {plateau }}\right)$ in the alkaline range. At high $\mathrm{pH}$ values all acidic surface functions are fully dissociated and, therefore, the polymer (composite) surface is negatively charged, lowering the $\mathrm{pH}$ causing the repression of the dissociation of the acidic surface groups, which will eventually cause a change in sign of the $\zeta$-potential caused by proton adsorption (Fig. 4). This is observed for at least PLDL and the TCP/PLDL composite. PLDL has an iep of 4.0 and a $\zeta_{\text {plateau }}$ of $-30 \mathrm{mV}$, which corresponds well to $\zeta$-potentials measured for PDLLA ${ }^{[46,45]}$ which have similar iep and $\zeta_{\text {plateau }}$ values. As expected the incorporation of inorganic fillers into the polymer matrix does effect the surface properties, i.e., the wettability and $\zeta$-potential, of the polymer. ${ }^{[47,48]}$ The incorporation of increasing loading fractions of T5 filler into PLDL results not only improved water wettability of the PLDL but also has a dramatic effect on the measured $\zeta$-potentials; the iep shifts toward lower $\mathrm{pH}$ values and in a steady decrease in the $\zeta_{\text {plateau }}$ values. In fact the iep could not be measured anymore for the composites containing the T5 powder. However, one should

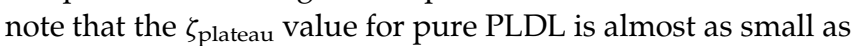
the one for the $40 \%$ glass/PLDL composite (Table 1). The measured $\zeta$-potentials of the films containing $40 \%$ titanium doped phosphate glass (T5) approach the values measured for commercially available $\beta$-TCP. ${ }^{[49]}$

\section{Mechanical Properties of Titanium Doped Phosphate Glass PLDL Composites}

The strain to failure of the filled polymers dropped significantly when compared with the pure polymer (Table 2). No significant differences were observed for both 
Table 2. Results of mechanical measurements. Young's modulus, ultimate tensile strength (UTS), and strain at failure detected in the polymer and filled polymer samples.

\begin{tabular}{lccl} 
& Young's modulus & UTS $\left[10^{8} \mathrm{~Pa}\right]$ & Strain [\%] \\
& {$\left[10^{9} \mathrm{~Pa}\right]$} & & \\
\hline PLDL & $2.4 \pm 0.76$ & $3.22 \pm 0.40$ & $77.00 \pm 5.23$ \\
PLDL-10\% TCP & $3.90 \pm 1.00$ & $2.0 \pm 0.4$ & $10.70 \pm 2.1$ \\
PLDL-10\% T5 & $1.94 \pm 0.90$ & $1.92 \pm 0.42$ & $23.40 \pm 13.88$ \\
PLDL-20\% T5 & $3.95 \pm 0.05$ & $1.64 \pm 0.38$ & $11.50 \pm 0.85$ \\
PLDL-40\%T5 & $4.51 \pm 0.91$ & $1.84 \pm 0.14$ & $12.57 \pm 3.07$ \\
& & &
\end{tabular}

filler types. The Young's modulus gradually increased with increase in the T5 filler loading fraction. The Young's modulus for PLDL filled with TCP was comparable to the modulus measured for the 20 vol\% of T5 filler. Further analysis of the tensile stresses in samples showed that for all filled samples the stresses decreased about 40-50\% compared with PLDL samples. Some differences were observed between both types of fillers; for $10 \mathrm{vol} \%$ TCP increase in Young's modulus was comparable to the one recorded for $20 \mathrm{vol} \% \mathrm{~T} 5$. No significant differences were observed for UTS and strain (with exception of $10 \mathrm{vol} \% \mathrm{~T} 5$ which showed twice as high stress level) for both types of fillers.

\section{Thermal Behavior of Titanium Doped Phosphate Glass/PLDL Composites}

Analysis of the differential scanning calorimetry (DSC) data showed that average onset and $T_{\mathrm{g}}$ for PLDL was 60.8 and $63.3^{\circ} \mathrm{C}$, respectively Fig. 5. Both evaluated temperatures were dropping gradually with increase in the content of the T5 filler. The drop was about 5\% for every increase in the glass filler content. No differences were observed between PLDL and TCP filled samples Fig. 5.

\section{Cell biology}

Cytoskeleton Evaluation: In the first instance surface area of the cells after 3 days in culture was assessed. Surface area is associated with number and size of the cells thus can give an indication of the cells adhesion and ability to spread on the surface at early time point. Surface area was calculated using

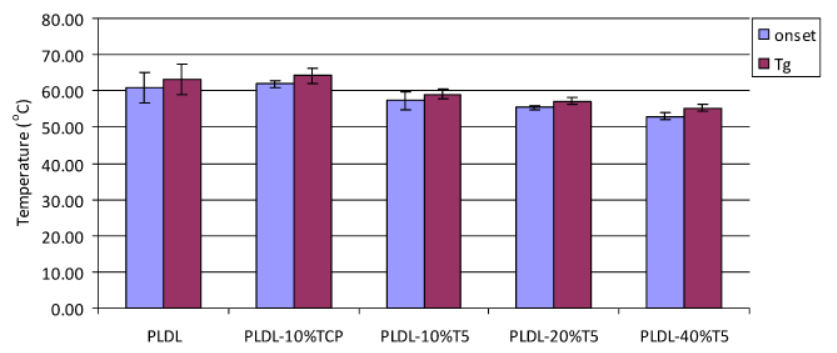

Fig. 5. Onset and glass transition temperature evidenced from DSC measurements for polymer and (titanium doped) phosphate glass polymer composites.

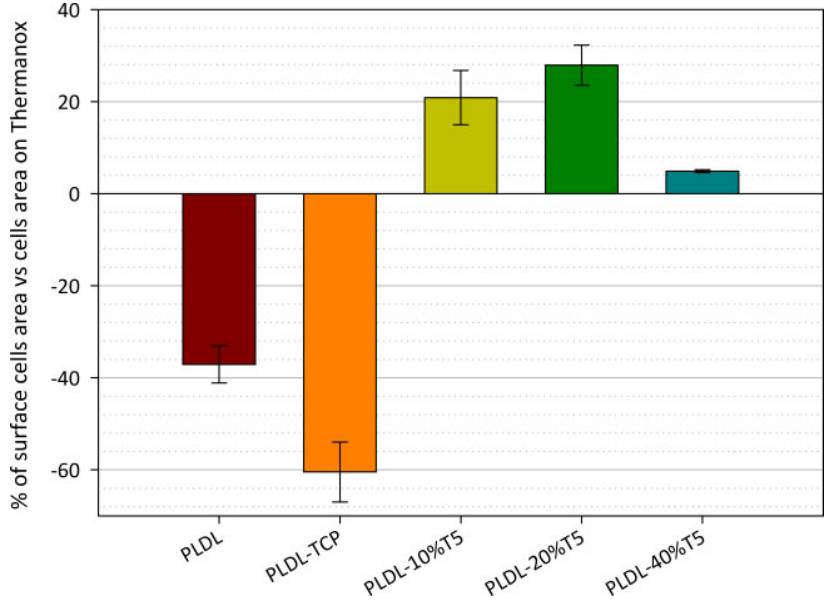

Fig. 6. Increase/decrease in total surface area of the cells that covered the samples in relation to the cells surface area on control Thermanox after 3 days in culture.

ImageJ software by outlining single cells and subtracting them from the image, then surface area of subtracted part was calculated and presented in a percentage of the total area of the whole image. Finally the calculated area was related to the area of the cells on control samples (Thermanox) indicating increase (positive values) or decrease (negative values) of cells area on the test samples versus control samples. This analysis revealed increase in total area of cells for the samples filled with T5 powder (Fig. 6). Increase was about 20 and 30\% for the samples with respectively 10 and $20 \mathrm{vol} \%$ of the filler. Further increase in the filler content to $40 \mathrm{vol} \%$ caused drop in the cells surface area, which was at the level observed for the control samples. For both PLDL and TCP filled samples drop of 38 and $60 \%$ of the total cells surface area was observed (Fig. 6).

Assessment of the cytoskeletons (actin, tubulin, and vinculin) organization revealed some differences between the samples (Fig. 7). For the pure polymer cells were rounded, occasionally elongated in one direction with well defined actin stress fibers and tubulin network [Fig. 7(a)]. However, both networks were organized mainly around the nuclei forming rather dense but not well spread structures. No focal adhesions were observed for the PLDL samples. Addition of $10 \mathrm{vol} \%$ of the T5 filler improved cytoskeleton organization and cell showed more developed structures with actin fibers (red) radiating in different directions and well organized tubulin (green) network [Fig. 7(b)]. Similarly to PLDL vinculin staining (green) evidenced no focal adhesions for PLDL-10\% T5. Increase in the T5 filler content (20 and $40 \mathrm{vol} \%$ ) did not change significantly organization of the cytoskeleton, which, however, was well organized and showed well defined actin and tubulin networks [Fig. $7(\mathrm{c}, \mathrm{d})$ ]. Some small and not very clear focal adhesions were evidenced for PLDL-40\% T5 samples. These structures were similar to the structures observed for the control positive samples. Nevertheless, it was observed that for the samples with the highest filler content there were a grater number of the cells with elongated structures in one 
Tubulin

Vinculin
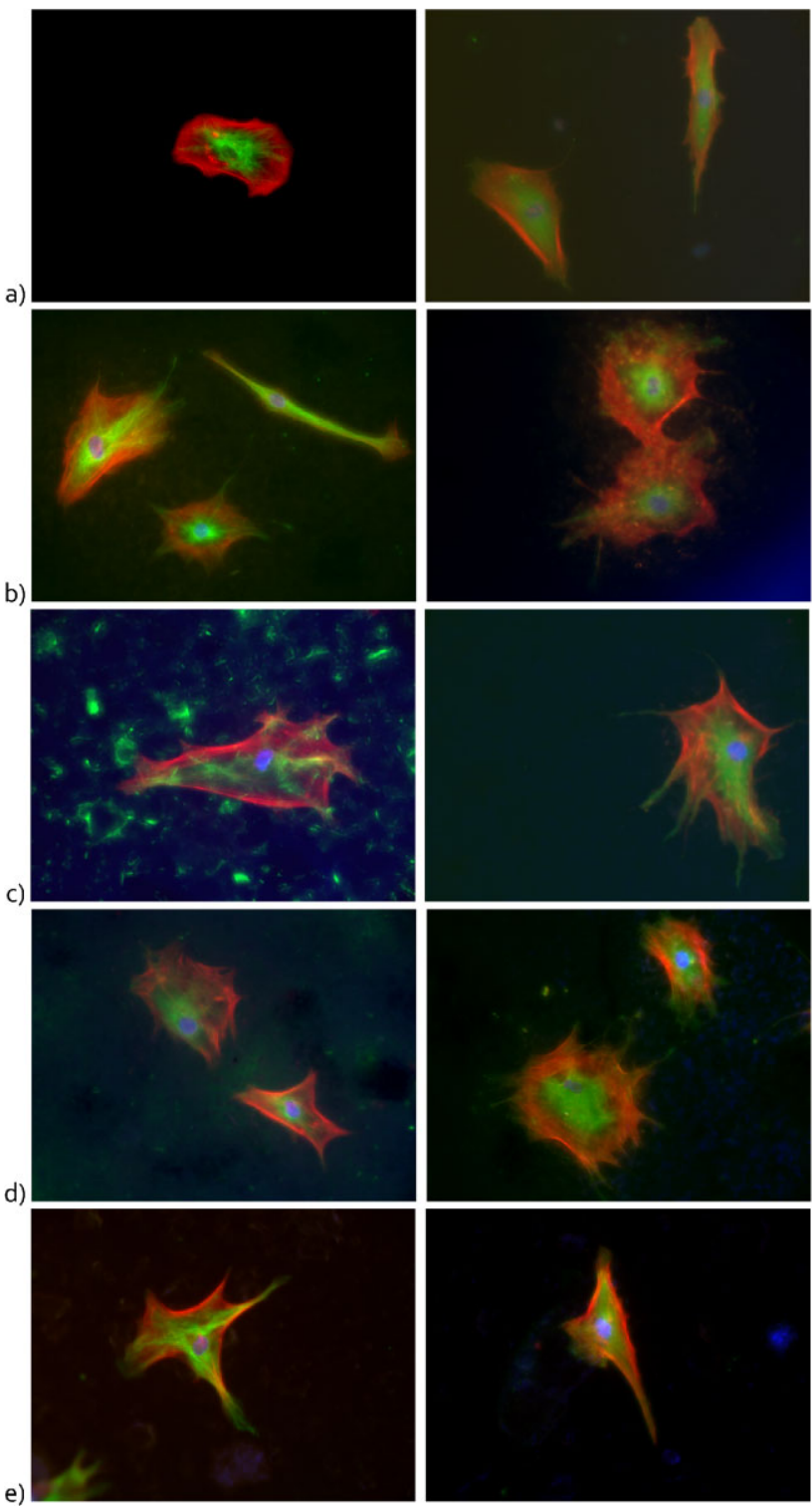

Fig. 7. Cytoskeleton and focal adhesion staining in human primary osteoblasts cultured on samples: (a) PLDL; (b) PLDL-10\%T5; (c) PLDL-20\%T5; (d) PLDL-40\%T5; (e) PLDL-TCP. Red-actin filaments; green-either tubulin microtubles (left images) or vinculin (right images) focal contacts; blue - cell nucleus.

direction, which could suggest specific topography or distribution of other cues (i.e., mechanical, chemical) on the surface and guiding cells in certain direction. Specific topography or particles orientation was, however, not confirmed on the SEM images. The samples with TCP filler cytoskeleton had also well organized tubulin network. The actin stress fibers were less developed and no focal adhesions were observed [Fig. 7(e)].

Expression of Osteocalcin and Osteopontin After 21 Days: Expression of the osteocalcin and osteopontin of the cells was assessed after 21 days in culture filled with 20 and $40 \mathrm{vol} \%$ of the T5 filler content supported osteoblastic differentiation particularly well (Fig. 8). Both osteocalcin and osteopontin
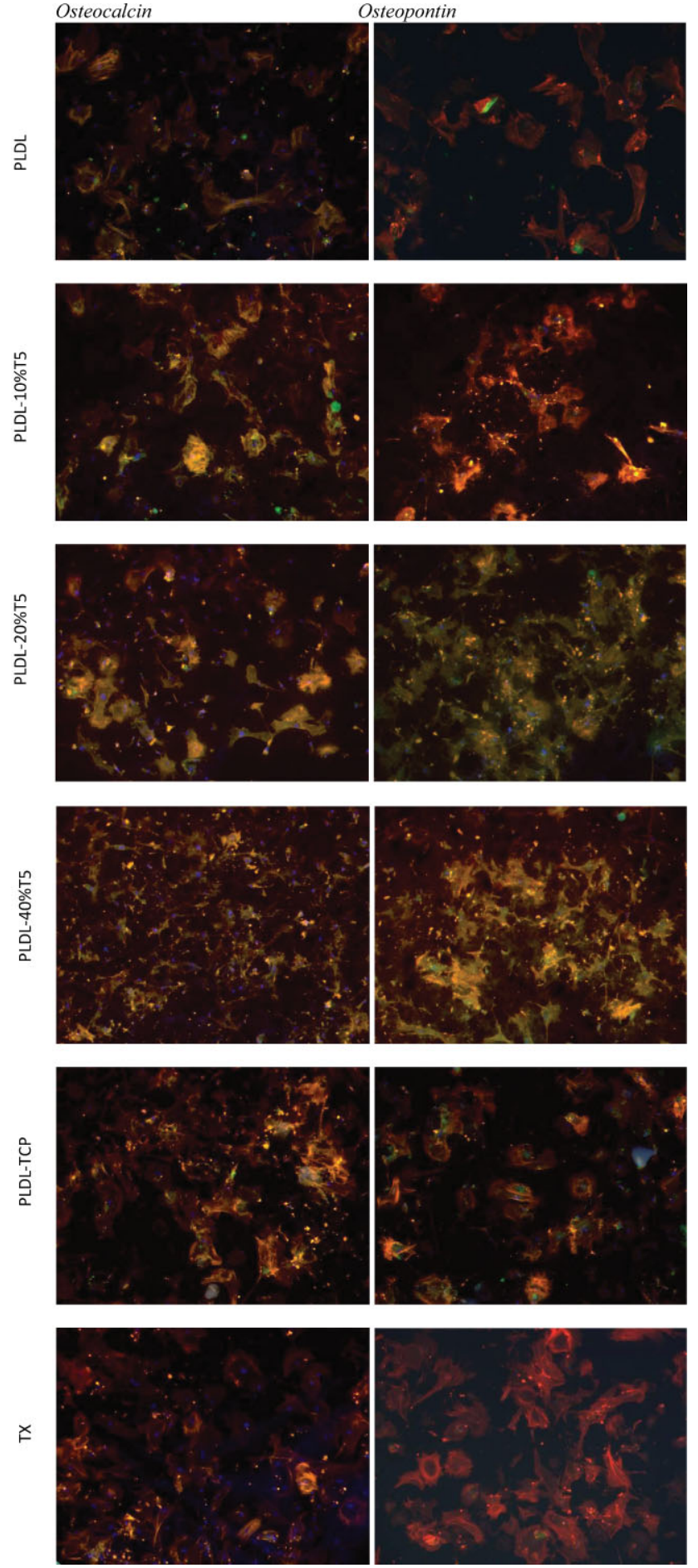

Fig. 8. Immunofluorescence images for the samples after 21 days in culture. Osteocalcin and osteopontin protein expression evidenced for the samples.

stainings were positive for all the samples. However, for the samples PLDL and PLDL-10\% T5 lower surface density of the cells was observed. In general samples filled with 20 and 40 vol\% of the T5 filler content supported osteoblastic differentiation particularly well. Nevertheless, both proteins were also expressed on TCP filled samples. 


\section{Discussion}

Currently development of biomaterials and attempts to improve materials bioactivity and biotolerability follow mainly two major approaches. ${ }^{[7,18,50]}$ First of them takes into account biochemical and biological interactions on the interface: device/tissue. This involves protein adsorption, cells adhesions, and differentiation, at nano- and micro-scale level. While the second approach correlates materials structure, morphology, topography, and other physical properties to the biological response. In both situations interactions at the biointerface are vital to obtain desired result. There is also consensus that developed biomaterials need to be biocompatible and biotolerated according to the newly proposed definitions (see Introduction). In this study authors hypothesis that with using tunable phosphate glass fillers for polymers, which are used as a biomaterial (e.g., membranes, implants, coatings), it is possible to stimulate human cells activity, improving their attachment, proliferation, and formability of the new bone. This is highly relevant for applications where enhancement of bone formation or osseointegration is desired (e.g., implants, scaffolds) and has a major importance to improve cell response to native polymer structures which are often passive. Therefore, it was assumed that use of biochemical cues such as phosphate glasses particles incorporated in the polymer matrix may be particularly useful to modulate the cells response (of not modified or patterned surface). It is believed that specific but tunable chemistry of the glasses is a key contributor to cell modulation. In turn this is a major advantage of such fillers in relation to other available fillers (e.g., TCP, HA).

In this study commonly used PLDL copolymer was used and filled with titanium doped phosphate glass powder (T5). Additionally, as a control $\beta$-TCP filler was used (TCP).

In general distribution of the T5 filler, which had non uniform size and angular shape, showed even distribution when concentration was at and above $20 \mathrm{vol} \%$. Uniform structure was also observed for TCP filler, which also had angular shape but uniform size about $12 \mu \mathrm{m}$ (according to the producer data). For all the samples in general good integration of the filler in the polymer was observed. Nevertheless some surface voids were observed for T5 filled samples. This was a result of the filler particles size which was not uniform and occasionally reached about $90 \mu \mathrm{m}$. Areas between those particles formed craters and due to slower evaporation resulted in a form of empty spaces that had surface character.

Wettability of materials is to certain extend related with biological and microbiological response, for this reason water contact angle and $\zeta$-potentials were measured. PLDL is rather hydrophilic hydrophobic. No change of the water wetting behavior was observed when TCP was incorporated into the PLDL. When, titanium doped phosphate glass filler was incorporated into PLDL the water wettability increased significantly with increase in filler content. Importantly, it was observed that for the T5/PLDL composites containing 20 and $40 \mathrm{vol} \%$ glass water wicked into the composites because of the intrinsically hydrophilic nature of the glass surface. Once the advancing motion of the droplet stopped the contact angle for the composites with 20 and $40 \mathrm{vol} \%$ rapidly (the faster the higher the glass content) decreased to zero. The water wicked into the gap at the interface between the glass and the polymer [Fig. $3(b, c)]$. The better wettability of the composite surface containing titanium doped phosphate glass is due to the hydrophilic nature of the glass filler.

The different wettability for both filler types, $\beta$-TCP and titanium doped phosphate glass, is due the larger particle size and different particles morphology of the T5 filler; the bigger T5 particle sediment faster to the bottom of the film during casting, which was the surface of the film that was characterized, and in turn this increased concentration of the filler on that side. The irregular particle size will have affected the packing of the particles within the composite. This may not have been the case for TCP filler.

The incorporation of increasing loading fractions of $\mathrm{T} 5$ filler into PLDL results not only in improved water wettability of the PLDL but has also a dramatic effect on the measured $\zeta$-potentials; the iep shifts toward lower $\mathrm{pH}$ values and in a steady decrease in the $\zeta_{\text {plateau }}$ values. Both, the shift of the iep and the decrease in $\zeta_{\text {plateau }}$ values for the titanium doped phosphate glass PLDL composites, are due to fact that more glass particles and, therefore, more strongly acidic functional groups, namely phosphates, which remain charged over a wide $\mathrm{pH}$ range, and (hydr)oxo groups $\left(\mathrm{TiO}(\mathrm{H})\right.$ and $\mathrm{Ti}_{2} \mathrm{O}(\mathrm{H})$ of titania in contact with water are present on the surface of the composites. However, the $\zeta_{\text {plateau }}$ value for pure PLDL is almost as small as the one for the PLDL containing $40 \mathrm{vol} \%$ titanium doped phosphate glass. PLDL contains few dissociable carboxylic acid surface groups, namely the end groups of a chain, but it is rather hydrophobic $\left(\theta=80^{\circ}\right)$ so its $\zeta$-potential is caused by the dissociation of carboxylic acids and the adsorption of ions in the electrochemical double layer. However, as soon as significant amounts, $10 \mathrm{vol} \%$, of T5 powder are incorporated into the PLDL it seems that the formation of the electrochemical double layer is solely determined by the strong dissociation of the stronger acidic surface groups present in the glass. The T5 particles are larger than the TCP particles and will, therefore, more rapidly sediment to the bottom of the cast film leaving them exposed on the film surface after preparation. The measured $\zeta$-potentials of the films containing $40 \mathrm{vol} \%$ titanium doped phosphate glass approach the values measured for $\beta$-TCP. ${ }^{[49]}$

Incorporation of the fillers into polymer matrix decreased ultimate tensile strength (UTS) but at high loading fractions resulted in a small increase in the Young's modulus; the reduction in tensile strength was comparable for both $\mathrm{T} 5$ and TCP fillers (for the same filler loading fraction). The filler in such case acts as a void inside the system and is considered as a weakening point because there are no chemical interactions between the filler and the matrix. However, there might be strong van der Waals interaction or even H-bonds. In general the strain to failure and tensile stress decreased substantially 
and were at similar level for all the filled samples. The Young's modulus was increasing with increase in the filler content. Importantly expected change to the mechanical properties observed after incorporation of TCP was observed after incorporation of the twice as much of the glass filler. This indicates that from mechanical point of view we could expect some differences between both types of fillers which depend on filler content. Therefore substituting TCP with titanium doped phosphate glass (T5) the mechanical properties will be altered for the same fillers concentration.

When glass transition temperature was analyzed it was found that $T_{\mathrm{g}}$ decreased by about $5 \%$ with every $10 \%$ increase in the T5 filler content. However, when TCP was considered insignificant increase in $T_{\mathrm{g}}$ was observed. Nevertheless, $T_{\mathrm{g}}$ temperatures were above $50{ }^{\circ} \mathrm{C}$ which is far from body temperature, thus no changes to the structure may be expected for those materials in body conditions due to temperature.

Finally and the most importantly human cells response to the materials was evaluated. Initial evaluation of the cells spreading on the basis of cell surface area in relation to the cells area on control samples revealed major differences between samples. The total surface area of the cells increased after adding T5 filler. Significant increase was observed for the filler concentration up to $20 \mathrm{vol} \%$. For the $40 \mathrm{vol} \%$ cells area was comparable with control Thermanox (TX) samples. Drop of the cells area for the highest filler concentration may be associated with various factors such as topography and morphology as well as surface chemistry, which determines both the wettability and $\zeta$-potential. However, from the analysis of the data from this study it may be hypothesized that increased modulus of the samples, thus stiffer substrate as long as good dynamic wettability and the highest $\zeta$-potentials could result in drop of the cells ability to spread. Nonetheless, cell response on the surface is multi factorial phenomena and requires further investigation which is ongoing. Unexpectedly, cells area dropped significantly for TCP filled samples. This is not fully understand and not in agreement with previous studies which however did not show relative cell growth, thus can not be directly compared. ${ }^{[21,25,51,52]}$

Cytoskeleton evaluations showed that incorporation of both fillers improved its organization. In general, for the filled samples cytoskeleton was well organized with well developed actin and tubulin networks when compared with pure polymer samples. Some small and not very clear focal adhesions were observed only for samples 40 vol\% glass filler. It can be concluded that filler improved organization of the cytoskeleton. Improvement was particularly well notable for the polymer filled up to $20 \mathrm{vol} \%$ of the T5 powder. These results are in agreement with cells area experiments. However, it was rather unexpected that the cells area for PLDL-TCP samples was significantly lower that for the other test samples. It is speculated that morphology of the filler, higher contact angles resulted in initial lower cells activity on the surface. Another explanation could be that due to the size of the particles on the top part of the samples thin polymer layer was created covering TCP thus its influence on cells was also hidden.
Further experiments were focused on ability of the samples to trigger osteoblastic differentiation and encourage mineralization of the cells. For all the samples both osteocalcin and osteopontin staining were positive, which indicate that either types of the filler could support cells maturation and osteoblastic differentiation. Nevertheless, positive staining was also observed for pure polymer and control samples. It can be concluded that fillers did not particularly influenced proteins expression, however further qualitative tests are required to reveal differences between the samples. These results are important as they demonstrated that the samples could support osteoblastic differentiation, thus may be potentially useful in bone tissue applications. Furthermore, detailed visual analysis of the samples reveled that density of the cells was lower for the following samples: pure PLDL, PLDL-10 vol\%T5, and PLDL/TCP, when compared with other samples, which may indicate that higher concentrations of the filler are more favorable to improve cells behavior.

In summary, it can be stated that hypothesis of the study was confirmed and using bioactive glass fillers can modulate cell response. It is assumed that biochemical cues related to the T5 filler composition were vital to improve cell response. Analyzed glass filler type altered mechanical properties of the final material at lower level as observed for tested $\beta$-TCP filler. These results pave the way for further studies where other types of glass dopands (including antimicrobial), different morphology and particles size may be consider to tune tissue response. Phosphate glasses as a fillers or composite components (e.g., fibers) hold a great promise in biomaterials and tissue engineering due to their potential to modulate bioresponse via chemistry, structure, degradation rate, and mechanical properties.

\section{Conclusions}

On the basis of the studies it can be concluded that:

- titanium doped phosphate glasses used as a filler for PLDL copolymers caused increase in wettability with increase in filler content,

- increase in the glass filler content caused increase in modulus value but a drop in stresses and strains, which lower then observed for TCP filler,

- mechanical properties observed for materials with $\beta$-TCP filler $(10 \mathrm{vol} \%)$ used as a control were similar to the materials with twice higher content of the glass filler (20 vol\%),

- glass transition temperature decreased with increase in the glass filler content,

- cells spreading improved for titanium doped phosphate glasses when compared with control, PLDL and $\beta$-TCP filled samples. The significant increase was observed for the glass filler content up to $20 \mathrm{vol} \%$,

- organization of the cytoskeleton improved for all filled samples (both types of filler) when compared with pure PLDL samples. The improvement was the most 
pronounced for samples with 20 and $40 \mathrm{vol} \%$ of the glass filler,

- there were no differences in mineralization (osteocalcin and osteopontin proteins) expression for all tested samples. However, greater filler concentrations showed more favorable results and greater cell density.

In the proposed approach authors tried to accomplish improvement and control of the cells response, which in turn may be translated onto controlled tissue regeneration and in further developments also controlled inflammatory response.

Our future research will include studies on the influence of morphology and size of the particles in nano-scale and their specific distribution in a polymer structure, as well as investigation of other dopands to inhibit antimicrobial properties.

Received: December 30, 2009

Revised: March 21, 2010

[1] L. L. Hench, J. Am. Ceram. Soc. 1991, 74, 1487.

[2] D. F. Williams, Biomaterials 2008, 29(20), 2941.

[3] E. A. Abou Neel, I. Ahmed, J. J. Blaker, A. Bismarck, A. R. Boccaccini, M. P. Lewis, Acta Biomater. 2005, 1(5), 553.

[4] I. Ahmed, C. A. Collins, M. P. Lewis, I. Olsen, J. C. Knowles, Biomaterials 2004, 25(16), 3223.

[5] F. R. A. J. Rose, R. O. C. Oreffo, Biochem. Biophys. Res. Commun. 2002, 292(1), 1.

[6] L. S. Nair, C. T. Laurencin, Prog. Polym. Sci. 2008, 32(8-9), 762.

[7] K. D. Jandt, Adv. Eng. Mater. 2007, 9(12), 1035.

[8] R. Liu, B. R. Saunders, J. Colloid Interface Sci. 2009, 338(1), 40.

[9] F. J. Xu, K. G. Neoh, Kang ET. Prog. Polym. Sci. 2009, 34(8), 719.

[10] R. Jayakumar, K. P. Chennazhi, R. A. A. Muzzarelli, H. Tamura, S. V. Nair, N. Selvamurugan, Carbohydr. Polym. 2010, 79(1), 1.

[11] A. C. Vieira, R. M. Guedes, A. T. Marques, J. Biomech. 2009, 42(15), 2421.

[12] H. Cao, N. Kuboyama, Bone 2010, 46(2), 386.

[13] L. Liu, W. Cai, Mater. Lett. 2009, 63(20), 1656.

[14] H. Tan, C. R. Chu, K. A. Payne, K. G. Marra, Biomaterials 2009, 30(13), 2499.

[15] X. Han, J. Pan, Biomaterials 2009, 30(3), 423.

[16] A. M. Young, S. M. Ho, E. A. bou Neel, I. Ahmed, J. E. Barralet, J. C. Knowles, Acta Biomater. 2009, 5(6), 2072.

[17] M. Wang, Biomaterials 2003, 24(13), 2133.

[18] S. Ramakrishna, J. Mayer, E. Wintermantel, K. W. Leong, Compos. Sci. Technol. 2001, 61(9), 1189.

[19] S. Higashi, T. Yamamuro, T. Nakamura, Y. Ikada, S. H. Hyon, K. Jamshidi, Biomaterials 1986, 7(3), 183.

[20] M. Wang, W. Bonfield, Biomaterials 2001, 22(11), 1311.
[21] Zigang, Ge, Lishan, Wang, Boon, Chin Heng, Tian, X-F, Kai, Lu, Tai, Weng Fan V, et al. J Biomater Appl 2009, 23(6), 533.

[22] J. Wei, Q. Z. Chen, M. M. Stevens, J. A. Roether, A. R. Boccaccini, Mater. Sci. Eng. C 2008, 28(1), 1.

[23] X. B. Yang, D. Webb, J. Blaker, A. R. Boccaccini, V. Maquet, C. Cooper, Biochem. Biophys. Res. Commun. 2006, 342(4), 1098.

[24] S. K. Misra, S. E. Philip, W. Chrzanowski, S. N. Nazhat, I. Roy, J. C. Knowles, J. R. Soc. Interface 2009, 6(33), 401.

[25] E. Vorndran, M. Klarner, U. Klammert, L. M. Grover, S. Patel, J. E. Barralet, Adv. Eng. Mater. 2008, 10(12), B67.

[26] I. Mehdawi, E. A. Abou Neel, S. P. Valappil, G. Palmer, V. Salih, J. Pratten, Acta Biomater. 2009, 5(7), 2525.

[27] E. A. Abou Neel, T. Mizoguchi, M. Ito, M. Bitar, V. Salih, J. C. Knowles, Biomaterials 2007, 28(19), 2967.

[28] E. A. Abou Neel, J. C. Knowles, J. Mater. Sci. Mater. Med. 2008, 19(1), 377.

[29] E. A. Abou Neel, W. Chrzanowski, J. C. Knowles, Acta Biomater. 2008, 4(3), 523.

[30] H. M. Aydin, Y. Yang, T. Kohler, A. El Haj, R. Muller, E. Piskin, Adv. Eng. Mater. 2009, 11(8), B83.

[31] S. Ponader, H. Brandt, E. Vairaktaris, C. von Wilmowsky, W. Gothel, R. Lutz, Adv. Eng. Mater. 2009, 11(8), B89.

[32] S. P. Valappil, D. Ready, E. Abou Neel, D. M. Pickup, W. Chrzanowski, L. A. O'Dell, Adv. Funct. Mater. 2008, 18(5), 732.

[33] S. P. Valappil, D. Ready, E. A. bou Neel, D. M. Pickup, L. A. O'Dell, W. Chrzanowski, Acta Biomater. 2009, 5(4), 1198.

[34] M. F. Gorriti, J. M. P. Lopez, A. R. Boccaccini, C. Audisio, A. A. Gorustovich, Adv. Eng. Mater. 2009, 11(7), B67.

[35] R. Shah, A. C. M. Sinanan, J. C. Knowles, N. P. Hunt, M. P. Lewis, Biomaterials 2005, 26(13), 1497.

[36] H. W. Kim, G. Georgiou, J. C. Knowles, Y. H. Koh, H. E. Kim, Biomaterials 2004, 25(18), 4203.

[37] I. Ahmed, C. A. Collins, M. P. Lewis, I. Olsen, J. C. Knowles, Biomaterials 2004, 25(16), 3223.

[38] F. Pernot, P. Baldet, F. Bonnel, J. Zarzycki, P. Rabischong, Ceram. Int. 2010, 9(4), 127.

[39] A. Alani, J. C. Knowles, W. Chrzanowski, Y. L. Ng, K. Gulabivala, Dent. Mater. 2009, 25(3), 400.

[40] T. Kasuga, M. Nogami, M. Niinomi, Adv. Eng. Mater. 2003, 5(7), 498.

[41] E. A. Abou Neel, W. Chrzanowski, S. P. Valappil, L. A. O'Dell, D. M. Pickup, M. E. Smith, J. Non-Cryst. Solids 2009, 355(16-17), 991.

[42] A. Curtis, M. Riehle, Phys. Med. Biol. 2001, 46(4), R47.

[43] M. J. Dalby, Med. Eng. Phys. 2005, 27(9), 730.

[44] K. Burridge, M. ChrzanowskaWodnicka, Annu. Rev. of Cell Dev. Biol. 1996, 12, 463. 
[45] L. Safinia, N. Datan, M. Höhse, A. Mantalaris, A. Bismarck, Biomaterials 2009, 26, 7537.

[46] L. Safinia, J. J. Blaker, V. Maquet, A. R. Boccaccini, A. Mantalaris, A. Bismarck, e-polymers 2005, 010.

[47] R. Verdejo, G. Jell, L. Safinia, A. Bismarck, M. M. Stevens, M. S. P. Shaffer, J. Biomed. Mater. Res. 2007, $874,632$.

[48] G. Jell, R. Verdejo, L. Safinia, M. S. P. Shaffer, M. M. Stevens, A. Bismarck, J. Mater. Chem. 2008, 18, 1865.
[49] M. A. Lopes, F. J. Monteiro, J. D. Santos, A. P. Serro, B. Saramago, J Biomed. Mater. Res. 1999, 45, 370.

[50] M. Geetha, A. K. Singh, R. Asokamani, A. K. Gogia, Prog. Mater. Sci. 2009, 54(3), 397.

[51] W. C. Xue, J. Biomed. Mater. Res, Part B-Appl. Biomater. 2009, 91(2), 831.

[52] M. Bohner, R. Luginbuhl, C. Reber, N. Doebelin, G. Baroud, E. Conforto, Acta Biomater. 2009, 5(9), 3524 . 